\title{
Immigration Estudiantine Tchadienne Et Incidence Sur La Vie Universitaire: Cas Des Étudiants Tchadiens De l'Université De Maroua
}

\author{
Luc Stéphane Massoma, PhD* \\ Université de Maroua, Cameroon
}

*Corresponding Author: Luc Stéphane Massoma, PhD Université de Maroua, Cameroon

\begin{abstract}
Chadian student immigration is a phenomenon that has been growing year after year in the city of Maroua since the creation of the University of Maroua. From the perspective of deep sociology, this article draws on data provided by direct observation and interviews to understand the various reasons and effects of this phenomenon on these populations in the reception area that is theirs. It presents the objective and subjective elements that motivate the migration projects of young Chadians oriented towards Cameroon. It focuses on the socio-cultural and other characteristics that, in the Far North of Cameroon, favor a smooth integration of these international students in this region of Cameroon. It helps to understand why the university, end even the city of Maroua are so attractive for Chadian students. Finally, it describes the living conditions of the students as they represent them themselves. This article gives access to a rather in-depth understanding of Chadian immigrant students in their representations of Cameroon, university training in Cameroon, and their living conditions amid their Cameroonian hosts.
\end{abstract}

Keywords: student immigration, Chadian students, university life, Maroua.

\section{INTRODUCTION}

Le lien entre la question de migration et celle d'éducation a été largement démontré dans des travaux d'origines disciplinaires diverses. Ces deux objets s'influencent réciproquement, puisque les migrations éducatives, qui ne sont pas de façon absolue distinctes des migrations économiques, sont parmi les plus récurrentes. Plusieurs problématiques et réalités sont associées autant à la variable éducative qu'à la variable migratoire, à l'instar du phénomène de «fuite des cerveaux », ou encore des dynamiques associées aux bourses d'études, de recherche ou de formation. Ces dernières qui sont des objets privilégiés pour le cadre des Sciences de l'éducation impliquent souvent des déplacements de durées variables ou, parfois, l'installation dans des statuts définitifs d'immigrants. C'est ainsi qu'au Cameroun, plusieurs jeunes arrivent chaque année d'autres pays pour s'inscrire dans les institutions éducatives et de formation professionnelle disponibles.

Les immigrants pour cause d'études au Cameroun se retrouvent à divers niveaux d'études et de formation et dans toutes les régions, mais surtout dans les régions contenant des villes universitaires. L'expérience de la ville de Maroua dans l'Extrême-Nord du Cameroun montre que souvent les diasporas étrangères dans leurs composantes estudiantines proviennent du pays frontalier le plus proche géographiquement et culturellement. Ainsi on constatera un afflux d'étudiants d'origine tchadienne dans les différents établissements de l'Université de Maroua. S'il est admis que les populations issues des diasporas sont généralement influencées par le milieu d'accueil auquel elles sont appelées à se conformer pour assurer leur adaptation et leur intégration, il est aussi vérifié qu'elles ont une influence non négligeable sur la vie des populations autochtones dans des domaines variés. On peut donc comprendre in extenso qu'un nombre important d'étudiants étrangers dans une université donnée ne peut évidemment être sans conséquence sur la vie universitaire. Le présent article s'intéresse ainsi à la mise en exergue desréalités qui sont celles des populations estudiantines d'origine tchadienne inscrites à l'Université de Maroua.

\section{Cadre Conceptuel Et Methodologie}

Les étudiants tchadiens de l'Université de Maroua sont les acteurs qui constituent notre public cible. Ils ont chacun intégré au cours du long parcours de la socialisation et des expériences diverses 
traversées des manières d'agir, de penser, de sentir qui leur sont inculquées de façon plus ou moins coercitive par des agents de socialisation institutionnels ou non. Le produit de ce processus d'inculcation est ce que Bourdieu a appelé habitus. Cet habitus est transporté par ces étudiants lorsqu'ils émigrent pour le Cameroun. Avec l'effectif de plus en plus croissant de ces étudiants dans l'université choisie comme site de l'étude, les éléments constituants de cet habitus s'insèrent dans le quotidien universitaire camerounais et y apportent des réalités pouvant être perçues de façon empirique.

Comme le relève Dubois (2014), «à force d'envisager l'immigration sous un angle chiffré, on occulte les raisons expliquant les flux migratoires $\gg$. Nous n'envisageons pas de produire au travers de cette recherche des statistiques sur l'immigration estudiantine tchadienne. Elle suivra par conséquent une démarche compréhensive. Il s'agit ici d'interroger les discours, les opinions et les usages des individus en vue d'accéder à la compréhension du pourquoi et du comment la mobilité estudiantine tchadienne s'opère, suivant quelles modalités et pour quels effets. La complexité et la sensibilité des données à recueillir à ce niveau rendent incontournable l'introduction d'une orientation qualitative dans notre recherche. C'est pour cette raison que l'observation directe est la principale technique de collecte des données ayant servi cette étude, sachant que nous avons des facilités liées à notre appartenance à l'institution concernée. Certaines données cependant sont le produit d'entretiens ayant permis d'enrichir cette étude. Les résultats présentés dans la suite sont donc le produit d'une recherche qualitative dans le sens qu'elle vise à produire et analyser des données descriptives telles que les écrits, les paroles et les comportements observables des personnes (Taylor et Bogdan, 1984).

\section{ConteXte Global}

L'Extrême-Nord est une des dix régions administratives du Cameroun. Elle a une superficie de 34246 $\mathrm{km}^{2}$ et est avec plus de 2720000 habitants la province dont la population est la plus dense (environ 80 habitants au $\mathrm{km}^{2}$ ). Elle a pour chef-lieu la ville de Maroua située trouve dans le département du Diamaré qui est l'un des six départements (avec le Mayo-Danay, le Mayo-Kani, le Mayo-Sava, le Mayo-Tsanaga et le Logone-et-Chari). L'arrivée de l'Université dans cette ville en 2009 y entraîne beaucoup de changements dans les mœurs, mais aussi rend la vie beaucoup plus chère. L'ExtrêmeNord est frontalier au Tchad et au Nigéria. C'est ce qui explique que cette région puisse être une destination particulièrement prisée par les voisins du Tchad qui partagent des traits culturels avec les habitants de ladite région. Une autre particularité de cette région est que ses habitants vivent pour la plupart dans une grande précarité. Ils sont victimes d'une paupérisation que les efforts déployés par plusieurs organismes internationaux partenaires du développement dans la région n'ont pu enrayer. D'autre part, l'Extrême-Nord est la zone la plus sous-scolarisée du pays. Le taux d'analphabétisme pour la population âgée de 15 à 24 ans y était il y a peu de 36,25\% pour les hommes et de 72,49\% pour les femmes (MINEDUC/DPRD/SDP, 2000). Avec la création d'une université dans la ville de Maroua, est venu un cosmopolitisme qui a contribué à modifier profondément cet environnement. Ainsi, cette ville est devenue un véritable pôle d'attraction pour des populations d'origines diverses. Cela a influencé tous ou presque tous les domaines de la vie dans cette ville, à l'instar des rapports interindividuels au quotidien, des transactions financières et d'affaires, des habitudes vestimentaires, etc. De touteévidence, le cadre éducatif qui est d'ailleurs le vecteur principal de ces changements ne pouvait en être exempt. Au fil du temps, les caractéristiques de la population estudiantine ont connu bien des modifications.

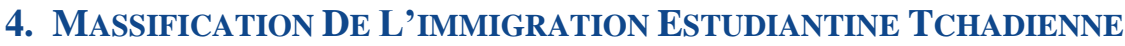

L'immigration estudiantine est une des plus répandues dans le monde. Il n'est donc pas étonnant de la retrouver dans la zone CEMAC. Cependant, le constat qui est fait est que dans les dynamiques migratoires au niveau de la sous-région, certains pays, notamment le Cameroun, sont plus des terres d'accueil et d'autres sont davantage des lieux de départ, à l'exemple du Tchad. On peut donc légitimement examiner la réalité de l'afflux des étudiants tchadiens dans les universités du NordCameroun et s'interroger sur les raisons qui poussent ces étudiants à quitter leur pays pour venir étudier au Cameroun. Comme le dit Bercque (1967), "Qu'il entre en tout cela de l'optimisme et de l'idéalisme, ou même quelque illusion, il serait » intéressant de le découvrir. 


\subsection{La Réalité D'une Massification De L'immigration Estudiantine Tchadienne A Maroua}

À sa création en 2008, l'Université de Maroua (UMa) n'était constituée dans les faits que d'un seul établissement, à savoir l'École normale supérieure de Maroua. Cette seule école supérieure visait la formation d'élèves enseignants et conseillers d'orientation pour davantage fournir le cadre de l'enseignement secondaire camerounais en personnel. L'entrée dans cette école et donc à l'UMa était alors conditionnée par la réussite à un concours pour les étudiants camerounais. À ce moment-là déjà, il y avait une présence tchadienne, même si elle n'était pas alors très importante. Les étudiants d'origine tchadienne pouvaient s'inscrire à l'ENS de Maroua à la suite d'une étude de dossier concluante avec avis favorable.

Au fil du temps, de nouveaux établissements ont été mis sur pieds" à l'Université de Maroua, à savoir :

- l'Institut supérieur du Sahel (I.S.S.) en 2010, qui devait devenir en 2017 l'Ecole Nationale Supérieure Polytechnique de Maroua (ENSPM);

- la Faculté des Sciences (F.S.) en 2013 ;

- la Faculté des Lettres et Sciences humaines en 2013, qui devait devenir quatre ans plus tard, à la faveur du transfert de la filière Beaux-Arts et Sciences du Patrimoine appartenant auparavant à l'ex-I.S.S., la Faculté des Arts, Lettres et Sciences Humaines (F.A.L.S.H.)

- la Faculté des Sciences juridiques et politiques en 2015

- la Faculté des Sciences économiques et de Gestion en 2015

- l'Institut des Mines et des Industries Pétrolières en 2015

Avec ces nouveaux établissements, la population estudiantine tchadienne a davantage augmenté, au point d'atteindre aujourd'hui plus de 6000 étudiants. Cette réalité peut s'expliquer par le fait que l'Université de Maroua est stratégiquement située sur le plan géographique par rapport au Tchad. Les étudiants tchadiens à Maroua viennent principalement des villes de Bongor, de Moundou et de Ndjamena. Les distances respectives entre Maroua et ces villes sont les suivantes d'après les données cartographiques 2019 de Google que nous avons comparé à ce que nous avons appris en entretien:

- Bongor - Maroua : $575 \mathrm{~km}$ pour 9h49minutes de route en voiture, mais si l'on considère le fait que majoritairement les gens font la traversée du Logone pour arriver à Yagoua et partir de là pour Maroua, cette distance se trouve réduite de plus de la moitié (Yagoua - Maroua : 115km pour $2 \mathrm{~h} 21$ minutes de route) contre $572,5 \mathrm{~km}$ pour $9 \mathrm{~h} 53$ minutes de route pour Bongor Ngaoundéré(ville ayant l'université la plus proche après Maroua)

- Moundou - Maroua : $593 \mathrm{~km}$ pour 9h54minutes de route en voiture, mais si l'on considère que ceux qui de Moundou viennent à Maroua passent généralement par Bongor, cette distance se trouve considérablement réduite (Bongor - Moundou : $234 \mathrm{~km}$ pour $3 \mathrm{~h} 21$ minutes de route). En traversant le Logone ils sont à Yagoua en 4h au plus, et à Maroua en 6h environ, contre $501 \mathrm{~km}$ pour 7h52minutes pour Moundou - Ngaoundéré

- Ndjamena - Maroua : 238,8 km pour $6 \mathrm{~h} 23$ minutes de route en voiture, contre $722 \mathrm{~km}$ pour 14h50minutes de route pour la distance Ndjamena - Ngaoundéré

Il ressort de cette évaluation des distances que l'Université de Maroua est beaucoup plus accessible que toute autre université pour l'étudiant tchadien moyen qui voudrait faire ses études dans une université camerounaise. Les universités camerounaises jouissent d'une bonne renommée auprès des étudiants d'origine tchadienne. Une répondante a dit : «On forme mieux ici (...). Quand on a 3 mois d'arriérés vous voulez qu'on enseigne bien comment? Ça ne peut pas être juste une question de snobisme... ». Elle suggère que les jeunes tchadiens en venant étudier au Cameroun sont convaincus d'y trouver une formation plus pointue que celle qu'ils auraient eue dans leur pays en pleine récession économique. Cet état de récession entraîne un traitement inapproprié des personnels enseignants qui

\footnotetext{
"Nous distinguons ici l'année d'émission du décret de création de l'établissement de l'année de lancement effectif des activités de l'établissement. C'est cette dernière qui nous intéresse ici.
} 
ne contribue pas à améliorer leurs performances et fragilise l'institution universitaire tchadienne. C'est là une autre raison qui justifie le grossissement des effectifs d'étudiants tchadiens à Maroua année après année. Nguirmanal (2017) présente des raisons données par des étudiants tchadiens pour expliquer leur désaffection pour les universités tchadiennes au profit des universités camerounaises, notamment celles de Ngaoundéré et de Maroua. Il écrit :

Beaucoup d'étudiants tchadiens partis étudier [auCameroun] expliquent leur choix par l'incapacité des universités tchadiennes à les accueillir et surtout, par l'élasticité de l'année académique. En l'occurrence l'année académique 2015 - 2016, dont le deuxième semestre n'a pas encore été abordé, par exemple à l'Université de N'Djamena.

Comme on le voit dans ce qui précède, la massification de l'immigration estudiantine tchadienne à Maroua est un fait. Elle peut également être le fait du bénéfice d'une coopération entre les universités des deux pays en question.

\subsection{Coopération Universitaire Cameroun-Tchad Comme Facteur D'immigration}

La coopération entre le Cameroun et le Tchad en tant que nations se répercute dans la coopération des universités tchadiennes avec les universités camerounaises, notamment celles du grand NordCameroun qui est directement frontalier au Tchad. Cette coopération transparaît dans les conventions existant entre ces universités à l'exemple de l'agrément de coopération entre l'Université de Maroua et l'Université de N'Djamena au Tchad. Celui-ci inclut notamment des échanges d'enseignants et d'étudiants, et implique également l'organisation conjointe des conférences, des séminaires et d'autres événements scientifiques et culturels. D'autres éléments matérialisent assez clairement l'importance de cette coopération. On peut citer la participation d'une délégation tchadienne aux jeux universitaires de 2019 à qui se sont déroulés à Maroua. La délégation tchadienne était composée d'étudiants de l'université de N'Djamena et d'autres étudiants de l'École normale supérieure de Bongor. Elle s'est illustrée par la présentation au public d'une parade inaugurale lors de la cérémonie d'ouverture de ces Jeux. La participation de ces athlètes aux jeux n'impliquait pas qu'ils figurent au classement, mais en venant observer et animer cet événement, ils offraient un symbole probant de la coopération universitaire tchado-camerounaise.

La possibilité prévue par la coopération inter-universitaire sus décrite d'être un étudiant tchadien boursier au Cameroun est en soi un élément qui peut déjà justifier l'attraction des universités camerounaises pour les jeunes tchadiens. D'autre part, lorsqu'ils font un rapport qualité-coût de la formation, les étudiants tchadiens estiment qu'ils sont gagnants en venant au Cameroun. Cela en raison de la réglementation en vigueur dans la zone CEMAC, qui fixe le montant des frais d'inscription dans les universités à 50.000 F CFA pour tous les étudiants ressortissants de la sousrégion. Alors, pour les mêmes frais d'inscription, ils peuvent avoir une scolarité plus stable et une formation de meilleure qualité de leur propre point de vue en s'inscrivant dans les universités camerounaises.

\subsection{Facteurs Socio-Culturels De L'immigration Estudiantine Tchadienne}

Certaines populations du grand Nord du Cameroun (qui comprend les régions de l'Adamaoua, du Nord et de l'Extrême-Nord) partagent des traits culturels avec les populations tchadiennes. Il y a par exemple des tribus que l'on retrouvera autant au Tchad qu'au Cameroun, notamment dans l'ExtrêmeNord. Certaines de ces tribus sont les suivantes : les Massa, les Moundang ou les Kera. Les membres de ces communautés parlent les mêmes langues qu'ils soient du Cameroun ou du Tchad ;ils partagent les mêmes cultures et, souvent, les mêmes mœurs et habitudes. Ils partagent également les mêmes traits physiologiques. Les Massa par exemple sont généralement d'une couleur noir foncé et de haute taille, qu'ils soient du Tchad ou du Cameroun. Ces similitudes socio-culturelles ont pour effet d'amener un tchadien à se fondre facilement dans le quotidien des populations camerounaises. Plusieurs grandes familles sont réparties des deux côtés de la frontière entre les deux pays. Du fait que l'on ne différencie pas à vue d'œil un tchadien d'un camerounais, les possibilités de stigmatisation et de xénophobie sont extrêmement réduites. Cela rend pour les tchadiens la vie assez agréable au Cameroun.

Le climat socio-culturel au Cameroun, et surtout dans le Nord, étant favorable aux immigrants tchadiens, on peut comprendre que les jeunes tchadiens puissent trouver attrayantes les études dans ce 
pays. Quand ils viennent à Maroua, ils changent d'environnement, mais ne sont pas dépaysés outre mesure. Plusieurs de ces étudiants ont une famille au Cameroun. Ils ne sont donc pas tout à fait étrangers. Ils peuvent échanger dans un idiome local avec des camerounais et se comprendre sans difficulté. On peut percevoir dans ce qui précède une autre raison justifiant l'intérêt des jeunes tchadiens pour les universités camerounaises, plus précisément pour celle de Maroua. L'afflux d'étudiants étrangers résultant de cet intérêt ne peut être sans effet sur les populations qui les accueillent, et plus précisément sur les réalités éducatives de l'Université de Maroua où ils s'inscrivent en grand nombre.

\section{IMMIGRATION ESTUDIANTINE TCHADIENNE : INCIDENCE SUR LA VIE UNIVERSITAIRE}

Plusieurs travaux réalisés dans des pays d'Afrique subsaharienne semblables au Cameroun ont montré qu'il y a une incidence des migrations sur le cadre global du développement dans ces pays. Il est établi que les migrations soutiennent ou altèrent ce que Furtado appelle «Le mythe du développement ». On pourrait donc penser à brûle-pourpoint qu'il est évident qu'il en est de même dans le contexte camerounais. Ce cadre global comprenant l'éducation comme un facteur majeur, on comprend que cette incidence n'épargne pas le domaine de l'éducation. Une immigration d'envergure ne peut se faire sans apporter de nouveaux éléments dans l'espace d'accueil. Ce sont ces éléments qui, au niveau de la vie universitaire, constituent les changements qui intéressent cet article.

\subsection{Diaspora Estudiantine Tchadienne Et Quête De Bien-Etre}

Selon l'AIDA*, le terme «diaspora »désigne : «la dispersion d'une communauté ethnique ou d'un peuple à travers le monde mais restant en relation [avec le pays de départ]».Selon Pascal Boniface, la «diaspora » désigne toute

population en situation de dispersion géographique, d'exil politique ou de migration économique [...] de façon stricte, une diaspora est le résultat d'un flux migratoire aboutissant à la constitution d'une population conservant une forte conscience de son identité (linguistique, religieuse...) et des rapports au moins affectifs avec son pays d'origine. (1996: 103).

Les migrations, quelles que soient les formes qu'elles revêtent, sont généralement associées à la recherche d'un mieux-être, à court, moyen ou long terme. Les diasporas se constituent donc autour de la quête de ce mieux-être que le pays d'accueil peut lui procurer de façon directe, ou qu'il peut lui donner les moyens d'acquérir. C'est dans ce second cas de figure que s'inscrivent la plupart des étudiants tchadiens immigrés au Cameroun.

En effet, la plupart des étudiants tchadiens avec lesquels nous avons eu contact planifient de prendre le diplôme convoité et de rentrer au Tchad où, semble-t-il, ils auront accès grâce à ce précieux sésame à un avenir radieux. Un étudiant tchadien de l'École normale supérieure nous a dit : "Comme je rentre là c'est pour être ministre », pour dire que le Master professionnel qu'il était sur le point d'obtenir lui donnerait accès à de très hautes fonctions dans son pays. La migration de retour fait donc partie des projets migratoires de la majorité des étudiants tchadiens inscrits dans les universités camerounaises, notamment à l'Université de Maroua.

Quelques-uns cependant, pour des raisons telles que la présence d'une famille au Cameroun, envisagent un avenir dans cette terre d'accueil. L'ascension fulgurante d'une personnalité telle que Gabriel Mbaïrobe est une source d'inspiration pour plusieurs parmi eux. En effet, c'est là un citoyen camerounais issu de l'immigration tchadienne qui, après avoir longtemps occupé des postes de cadre au sein d'une société importante sur le plan national, a été hissé au poste prestigieux de Ministre de l'agriculture. Son parcours laisse entrevoir la possibilité pour un étudiant tchadien de s'installer au Cameroun de façon définitive et de réussir à mener à terme un projet professionnel dans ce pays. Une telle personne pourrait offrir un avenir prometteur à sa progéniture qui jouira du statut de citoyen camerounais au même titre que les autres camerounais. Nous avons ainsi des familles tchadiennes à l'origine qui ont tout l'air aujourd'hui de familles camerounaises et dont les membres sont des agents de la fonction publique camerounaise, à l'instar de la famille Garoum avec laquelle nous avons eu à nous entretenir.

A partir de ce qui précède, on réalise que l'étudiant tchadien qui immigre au Cameroun le fait avec des perspectives d'enrichissement intellectuel certes, mais aussi avec en vue des carrières professionnelles engageantes, soit dans leur pays d'origine pour ceux d'entre eux qui comptent y

*Algerian International Diaspora Association 
rentrer, soit dans leur terre d'accueil pour ceux qui souhaitent s'y installer de façon définitive et y faire leur vie. L'ampleur de cette mobilité estudiantine engendre ce qu'on peut appeler d'après Martiniello et Rea de véritables «carrières migratoires », en ce sens que, compte tenu des objectifs visés par les étudiants tchadiens, elle inclut la notion de réussite ou d'échec du projet migratoire.

\subsection{Les Etudiants Tchadiens : Vie A l'Université De Maroua}

Quand on observe la vie des étudiants tchadiens de l'Université de Maroua on fait effectivement un certain nombre de constats. Tout d'abord, on constate chez ces étudiants un fort instinct grégaire. Ils ont en effet tendance à vivre ensemble et sont très solidaires les uns des autres. On les retrouve assemblés dans des chambres dont ils assument ensemble les coûts de location, d'eau et d'électricité. $\mathrm{Ce}$ fort communautarisme ne se manifeste pas seulement dans la vie péri-universitaire. En effet, ils sont souvent ensemble même sur le campus. Mais, contrairement aux membres d'une diaspora telle que la diaspora chinoise, on ne peut dire qu'ils ne se mêlent pas aux autres. Bien qu'ils communiquent avec les étudiants camerounais, ils passent généralement du temps ensemble. Deux jeunes camerounais avec lesquels nous nous sommes entretenus semblaient d'accord sur le propos suivant tenu par l'un d'eux : «Les tchadiens font tout ensemble. Quand un déménage les autres aussi. Quand un est quelque part les autres viennent $»$.

Sur un plan purement académique, les étudiants tchadiens ne rechignent pas à travailler avec les étudiants camerounais quand le besoin se trouve. Les relations entre ces étudiants immigrés et les étudiants camerounais sont assez cordiales. Si certains d'entre eux se distinguent par des prestations académiques positivement remarquables, la majorité de ces étudiants immigrés ont des performances en-deçà de la moyenne. Cela est peut-être dû aux causes que présente Nguirmanal (2017) :

Au lieu de se consacrer au but du déplacement, nombre d'entre ces étudiants s'adonnent à des activités peu conciliantes avec leurs études. «La plupart des étudiants tchadiens sont des fêtards. Et très peu finissent leur cursus sans reprendre à plusieurs reprises les niveaux, même si quelques rares font des efforts », explique un enseignant de Droit public à l'Université de N'Gaoundéré.

Pour illustrer le fait que plusieurs d'entre eux se plaignent de leurs conditions de vie, Nguirmanal continue en présentant lesdites conditions pour des étudiants tchadiens de l'Université de Ngaoundéré (voir encadré).

\section{Encadré. Conditions de vie des étudiants tchadiens au Cameroun}

Le coût de vie. En dehors des minis cités où un studio coûte entre 15.000 et $30.000 \mathrm{~F}$ CFA par mois, les parents déboursent à la fin du mois entre 30.000 et 50.000 FCFA pour la ration alimentaire. Pour une année scolaire, certains parents emploient entre 540.000 F CFA et 700.000 FCFA. Et pour les parents bien lotis, il faut environ 750.000 F CFA à 1.000.000 F CFA. Michaël, étudiant en Master 1 de Droit Privé à l'Université de N'Gaoundéré où il est inscrit depuis 2009, est issu d'une famille modeste. Michaël qui dit s'être lancé le défi de ne pas décevoir les siens explique recevoir de son père, un cadre diplomatique de son état, ce dont il a besoin pour étudier. « Le minimum pour une année scolaire que mon père m'envoie, pour la ration alimentaire et le loyer, est de 960.000 F CFA. Je paie le loyer à 30.000F CFA le mois et je reçois 50.000 FCFA par mois pour la ration alimentaire». Si sur le plan matériel et financier Michaël rencontre moins de difficultés, sur le plan académique, ce n'est pas facile. Car, dit-il, le système camerounais est plus rigoureux que le système tchadien. «Au début, j'ai trouvé l'écart trop grand, maintenant, je me suis adapté ». Michaël confie avoir repris trois fois. «Il faut reconnaître qu'il y a trop de laisser-aller chez nous. J'ai commencé à l'Université de N'Djamena avant de venir à N'Gaoundéré », conclut-il.

Escroqueries, maladies imaginaires ...Certains étudiants tchadiens expliquent pour leur part que leurs nombreux échecs sont dus aux difficultés sociales qu'ils rencontrent. Notamment, des difficultés liées à la nourriture. En cause, les nombreuses charges financières des parents. Pour faire face, plusieurs choisissent de se loger à deux dans une même chambre. Fait ainsi, ils partagent et supportent mieux les besoins. «Haroun était juste uneconnaissance au Tchad. Arrivé ici, nous nous sommes entendus pour partager une même chambre. Celui à qui les parents envoient de l'argent en premier fait des provisions en attendant que l'autre ne reçoive sa part », explique Moïse, qui vient de commencer la première année de Géologie. Dans ces conditions, le risque est gros de tomber sur des amis que Moïse qualifie d'escrocs. Ils usent de tous les moyens pour obtenir ce qu'ils veulent. Faux et usage de faux, escroquerie, abus de confiance, vol, etc. ces étudiants ont souvent pour cibles leurs propres compatriotes. D'autres encore, explique-t-il, inventent une maladie grave pour extorquer de l'argent à leurs parents juste dans le but de 
boire de l'alcool. Et ceux-là rentrent souvent bredouilles après plusieurs années passées à l'université.

«Concubinage ». Une autre catégorie d'étudiants tchadiens à N'Gaoundéré comme dans le reste des universités du Cameroun. Toujours pour question de se partager les charges estudiantines comme sus évoqué, ceux-ci se mettent en semble pour vivre une sorte de «concubinage ». Et comme on peut s'y attendre, les actes voluptueux prennent le dessus sur les études. Conséquences : beaucoup rentrent sans diplômes, malades ou pères et mères d'enfant.

Jean Nguirmanal,« Ce que font les étudiants tchadiens au Cameroun », in Le Tchadanthropus, 8 janvier 2017

\section{CONCLUSION}

L'observation dans une perspective sociologique des dynamiques qui sous-tendent l'immigration estudiantine tchadienne et des effets de celle-ci sur ceux qui en sont les acteurs est révélatrice de réalités assez complexes. Le présent article a de façon détaillée examiné les déterminants de l'attraction des universités camerounaises, et plus précisément de l'Université de Maroua, sur les étudiants tchadiens dontles effectifs augmentent régulièrement. D'autre part, il s'est attardé sur les incidences sur la vie universitaire de cette présence tchadienne à Maroua et sur les conditions de vie de ces étudiants immigrés. Il ressort de cet examen que si plusieurs facteurs concourent à rendre l'Université de Maroua attrayante pour ces étudiants et à provoquer leur venue, il ne demeure pas moins vrai qu'une fois à Maroua, ils ne sont pas forcément dans des conditions propices à un bon rendement académique. Dans certains cas, ils en sont eux-mêmes la cause, mais dans d'autres ils sont victimes de contingences indépendantes de leur volonté.

\section{BIBLIOGRAPHIE}

[1] Boniface, Pascal (dir.), 2016, Dictionnaire des relations internationales, Paris, Hatier.

[2] Bourdieu, Pierre, 1980, Le sens pratique, Paris, Editions de Minuit.

[3] Dubois, Xavier, 2014, «Les vraies raisons de la migration » in Contrastes_numéro 160, «Politique d'asile : la Belgique forteresse», Équipes Populaires, janvier-février 2014.

[4] Furtado, Celso 1984,Le mythe du développement économique, Paris, Anthropos.

[5] Martiniello, Marco et Rea, Andrea, 2011, « Des flux migratoires aux carrières migratoires », in SociologieS [En ligne], Dossiers, Migrations, pluralisation, ethnicisation des sociétés contemporaines, mis en ligne le 18 octobre 2011, consulté le 11 novembre 2019.URL : http://sociologies.revues.org/3694

[6] Nguirmanal Jean (2017), «Ce que font les étudiants tchadiens au Cameroun » in Le Tchadanthropus, Web-presse.www.letchadanthropus-tribune.com, mis en ligne le 8 janvier 2017 URL: https://www.letchadanthropus-tribune.com/tchadcameroun-ce-que-les-etudiants-tchadiens-font-aucameroun/

[7] TaylorSteven, BogdanRobert (1984), Introduction to qualitative research methods, $3^{\text {rd }}$ edition.

Citation: Luc Stéphane Massoma. "Immigration Estudiantine Tchadienne Et Incidence Sur La Vie Universitaire: Cas Des Étudiants Tchadiens De l'Université De Maroua” International Journal of Humanities Social Sciences and Education (IJHSSE), vol 8, no. 2, 2021, pp. 150-156. doi: https://doi.org/10.20431/23490381.0802014 .

Copyright: (C) 2021 Authors. This is an open-access article distributed under the terms of the Creative Commons Attribution License, which permits unrestricted use, distribution, and reproduction in any medium, provided the original author and source are credited. 